\title{
25 Research Square \\ Clinic Correlation and Prognostic Value of P4HB and GRP78 Expression in Gastric Cancer
}

Chenfei Zhang

Nantong Tumor Hospital https://orcid.org/0000-0001-6618-8892

Wenjing Zhao

Nantong Tumor Hospital

Xingsong Zhang

Nantong Tumor Hospital

Tongyu Wu

Nantong Tumor Hospital

Xunlei Zhang

Nantong Tumor Hospital

Li Song

Nantong Tumor Hospital

Weiwei Xu

Nantong Tumor Hospital

Xudong Chen

Nantong Tumor Hospital

Lei Yang ( $\nabla$ yanglei.53@163.com )

Nantong Tumor Hospital

Primary research

Keywords: P4HB, GRP78, gastric cancer, postoperative adjuvant chemotherapy, nomogram

Posted Date: June 22nd, 2020

DOI: https://doi.org/10.21203/rs.3.rs-36543/v1

License: (c) (i) This work is licensed under a Creative Commons Attribution 4.0 International License.

Read Full License 


\section{Abstract}

Background: Prolyl 4-hydroxylase, beta polypeptide (P4HB) and Glucose-regulated protein 78 (GRP78) are represent for poor prognosis of various cancers. However, rare research investigate the correlation of them in cancer. This study aimed to investigate the correlation and prognostic value of P4HB and GRP78 expression along with clinical features in gastric cancer (GC).

Methods: A total of $150 \mathrm{GC}$ tissue samples separately evaluated P4HB and GRP78 protein expression by immunohistochemistry. Association of P4HB and GRP78 expression with clinicopathological features was analyzed. Bioinformatics analyses were performed to find correlation between mRNAs and pathways. Kaplan-Meier analyses were taken to compare survival curves. Univariate and multivariate Cox regression models used to analyze the impact of prognostic factors on overall survival (OS). A prognostic nomogram was constructed based on the multivariate Cox regression model, and compared to TNM stage in discrimination ability and clinical usefulness.

Results: P4HB and GRP78 expression were both associated with tumor invasion and lymph node metastasis. P4HB protein positively correlated with GRP78, as well as the same tendency of mRNAs in both GC tissue and cell databases. Bioinformatics analyses indicated that P4HB and GRP78 may be associated with protein folding and response to ER stress, and the protein processing in ER pathway. High single or co-expression of P4HB and GRP78 represented for a shorter OS. When stratified by postoperative adjuvant chemotherapy, the high co-expression was only related to unfavorable prognosis of with chemotherapy group, especially in advanced stage. Multivariate Cox regression analysis identified co-expression of P4HB and GRP78 as an independent prognostic factor for OS. The nomogram including the co-expression was better than TNM stage in discrimination ability and clinical usefulness through the receiver operating characteristic (ROC) and decision curve analysis (DCA) curves.

Conclusion: P4HB was positively correlated with GRP78 expression, co-expression of them was an independent prognostic factor, could serve as a predictive marker for GC patients with postoperative adjuvant chemotherapy in advanced stage.

\section{Introduction}

Gastric cancer (GC) is the fifth common diagnosed cancer and the third most frequently cause of cancer death global wide, relating to over one million new cases and an estimated nearly zero point eight million deaths in 2018, along with the incidence rate is still markedly elevating in Eastern Asia [1]. Since the best treatment period of most GC patients had already missed when they clinically diagnosed, the prognosis of GC remains poor [2].The grim fact that survival trends were little increased in most countries between 1995-1999 and 2010-2014 disappoints us, despite efforts to improve treatment strategies [3].Owing to huge biological heterogeneity, even with similar clinical features, the prognoses of GC would perform great differences $[4,5]$. Therefore, precisely evaluate the prognosis of GC with the aid of better predictors is urgently needed. 
The endoplasmic reticulum (ER) is responsible for calcium dynamic balance, lipid biogenesis and participate in folding of either secretory or membrane bound proteins. Once various stresses such as hypoxia break its homeostasis, the resulting abnormal accumulation of unfolded and misfolded proteins together constitute ER stress [6].To reconstruction ER homeostasis, a series of signaling pathways named unfolded protein response (UPR) subsequently activates, which makes up of three main branches: 1) PERK (pancreatic ER kinase-like ER kinase, also known as elF2AK3) -elF2a (eukaryotic translation initiation factor 2a ); 2) ATF6 (activating transcription factor-6); 3) IRE1 (inositol-requiring protein 1, also known as ERN1)-XBP1 (X-box binding protein 1) [6-8]. Tumors trend to accompany with hypoxia, acidosis and nutrient deprivation, frequently induce ER stress, together with follow-up activation of UPR play core roles in tumorigenesis, proliferation and survival of cancer cells [6].

Prolyl 4-hydroxylase, beta polypeptide (P4HB, also known as PDIA1) belongs to protein disulfide isomerase (PDI) family, close connects with ER stress and UPR, as an enzymatic molecular chaperone to reconstruct misfolded proteins [9]. P4HB is proved to be upregulated in a variety of cancers, such as lung cancer [10], diffuse glioma [9], colon cancer [11]. As a result, P4HB has been reported by numerous researches to be a potential target for cancer therapy [12-14]. But studies referred to GC were rather limited. Only one recent research showed P4HB positively correlated with hypoxia-associated biomarkers, overexpression of P4HB indicated poor prognosis in GC patients [15].

Glucose-regulated protein 78 (GRP78, also known as HSPA5 or BiP) is also an ER chaperone protein, will dissociate from three specific sensors (PERK, ATF6 and IRE1) in response to ER stress, and subsequently actives UPR.6 [16]. General overexpression of GRP78 in different cancers including GC has been widely accepted, also GRP78 could serve as a good indictor of poor prognosis and contribute a lot to therapy resistance $[17,18]$.

Xia et al identified that P4HB through downregulating GRP78 promoted hepatocellular cancer tumorigenesis [19]. However, in GC, single overexpression of the two ER chaperone proteins has been reported to represent poor prognosis, meaning P4HB may not negative correlate with GRP78. Due to rare relevant research, we aimed to investigate the correlation between P4HB and GRP78 expression and clinical features, as well as their prognostic implications in GC. Furthermore, we integrated the expression of P4HB and GRP78 with other prognostic variables to predict overall survival (OS) after surgery in GC patients.

\section{Materials And Methods}

\section{Patients}

Amount to 150 GC patients who underwent gastrectomy from 2007 to 2013 at Affiliated Tumor Hospital of Nantong University (Nantong, China) were enrolled in our retrospective study. All patients confirmed histopathology diagnosis after gastrectomy, and none of them received preoperative chemotherapy or radiotherapy. Patients with incomplete follow-up data and histopathological diagnosed neuroendocrine 
or adenosquamous carcinoma were excluded from the cohort. The pathological tumor-node-metastasis (TNM) stage conformed to TNM Staging Manual (7th edition) from the AJCC (American Joint Committee on Cancer). Fluorouracil-based postoperative adjuvant chemotherapy were mainly performed in patients with advanced disease ( $\mathbb{\nabla} / \mathbb{Q} / \mathbb{T} \mathrm{TNM}$ stage). The clinicopathological parameters included were shown below: age, gender, Bormann type, tumor size, Lauren's classification, differentiation, histological type, depth of tumor invasion, lymph node metastasis, TNM stage, vessel invasion, perineural invasion and fluorouracil-based postoperative adjuvant chemotherapy (sTable 1). The median follow-up time was 68.0 (range 2.5-92.5) months. Overall survival (OS) was defined as the period between the date of gastrectomy or the death or the last follow-up. If patients ended follow-up in keeping alive, data were censored. The study had achieved the approval from the Ethics Committee of Affiliated Tumor Hospital of Nantong University and the informed consent of all in cohort patients.

\section{Immunohistochemistry (IHC)}

The tissue microarrays (TMA) were constructed by duplicate $1.5 \mathrm{~mm}$ formalin-fixed paraffin-embedded tissue cores of two different areas. We performed IHC according to the standard protocol for the immunostaining provided by a previous study [20]. P4HB expression used a mouse anti-P4HB monoclonal antibody (1:100, Abcam, Cambridge, UK). While GRP78 IHC used a rabbit anti-GRP78 monoclonal antibody (1:100, Abcam, Cambridge, UK). The mi-quantitative H-score, ranged from 0 to 300 , was derived from the staining intensities ( 0 : negative, 1 : weak staining, 2: moderate staining, 3: strong staining) and the proportion of stained ( $0-100 \%)$. The $\mathrm{H}$-score was evaluated independently by two experienced pathologists who were blind to clinical information. The median $\mathrm{H}$-score was used as the expression cutoff point (P4HB and GRP78 median H-score both $=200)$. Under the condition, for both proteins, $\mathrm{H}$-scores less than 200 were considered to low expression, while $\mathrm{H}$-scores on the opposite were considered to low expression, respectively.

\section{Bioinformatics analyses}

The Gene Expression Profiling Interactive Analysis (GEPIA, http://gepia.cancer-pku.cn/index.html) [21], which is an interactive web server based on The Cancer Genome Atlas (TCGA), was used to find the correlation of mRNAs. The mRNA expression data of totally 1168 cell lines, including $37 \mathrm{GC}$ cell lines, was downloaded from the Broad Institute Cancer Cell Line Encyclopedia (CCLE, https://portals.broadinstitute.org/ccle) [22]. Gene Ontology (GO) analysis and Kyoto Encyclopedia of Genes and Genomes (KEGG) analysis were performed by R package "clusterProfiler". The correlation coefficients of genes visualized in KEGG pathway maps were performed by R package "pathview".

\section{Statistical Analysis}

SPSS version 25.0 (IBM, Armonk, NY) was applied to for the $\chi 2$ test to explore the correlations among biomarker variables (P4HB and GRP78) and clinicopathological variables, the Spearman rank correlation 
to evaluate the correlation between $\mathrm{H}$-scores of P4HB and GRP78, the univariate and multivariate analyses by the stepwise Cox proportional hazard regression model to estimate the crude hazard ratios (HRs) and 95\% Cl of HRs. MedCalc Software version 19.0.4 (MedCalc, Mariakerke, Belgium) was applied to compare two correlation coefficients. The R packages "rms", "survival", "foreign", "survivalROC" and "survminer" were used for ploting the nomogram, the calibration curves to evaluate the calibration ability of the nomogram, the ROC curves to assess the discrimination ability of the nomogram, Kaplan-Meier curves to estimate prognosis and calculate the Harrell's C-index also to evaluate the discrimination ability of the nomogram. Decision curve analysis (DCA) curves were plotted by R package "stdca", whose source file was from www.mskcc.org, to analyze the clinical usefulness of the nomogram. Two-sided $P$ value less than 0.05 was considered statistically significant.

\section{Results}

\section{Association of P4HB and GRP78 protein expression with clinicopathological characteristics in GC}

We detected the expression of P4HB and GRP78 separately by immunohistochemistry (IHC) in $150 \mathrm{GC}$ tissues. The representative images of P4HB and GRP78 staining are shown in Fig. 1. A total of 74 patients (49.3\%) were P4HB low expression and 76 patients (50.7\%) were P4HB high expression, as for GRP78 expression, there were 71 low cases $(47.3 \%)$ and 79 high cases $(52.7 \%)$ respectively. Furthermore, the expression of $P 4 H B$ was correlated with age $(P=0.021)$, Bormann type $(P=0.029)$, depth of tumor invasion $(P=0.001)$, lymph node metastasis $(P=0.018)$, postoperative adjuvant chemotherapy $(P=$ $0.008)$, while GRP78 expression was only correlated with depth of tumor invasion $(P=0.041)$ and lymph node metastasis $(P=0.043)$ (Table 1$)$. And P4HB expression was strongly correlated with GRP78 $(P<$ 0.001). Subsequent Spearman rank correlation analysis of P4HB and GRP78 H-score proved there existed a significant positive correlation $(R=0.587, P<0.001)$ (sFigure $1 A)$. By investigating the correlation coefficients of different clinicopathological characteristics, the correlation coefficients were significantly elevated in tumor size $\geq 5 \mathrm{~cm}(P=0.037)$, TNM $\otimes / \otimes$ stage $(P=0.037)$ and positive vessel invasion $(P=$ 0.049 ) subgroups, as shown in sTable 2. 
Table 1

Relationship between P4HB/GRP78 protein expression and clinicopathological characteristics in GC patients

\begin{tabular}{|c|c|c|c|c|c|c|c|c|}
\hline \multirow{2}{*}{$\begin{array}{l}\text { Clinicopathological } \\
\text { parameters }\end{array}$} & \multicolumn{4}{|c|}{ P4HB expression } & \multicolumn{4}{|c|}{ GRP78 expression } \\
\hline & Low & High & $x^{2}$ & $\begin{array}{l}P \\
\text { value }\end{array}$ & Low & High & $x^{2}$ & $\begin{array}{l}P \\
\text { value }\end{array}$ \\
\hline & 74 & 76 & & & 71 & 79 & & \\
\hline Age(year) & & & 5.369 & 0.021 & & & 2.404 & 0.121 \\
\hline$<65$ & 48 & 35 & & & 44 & 39 & & \\
\hline$\geq 65$ & 26 & 41 & & & 27 & 40 & & \\
\hline Gender & & & 0.408 & 0.523 & & & 0.008 & 0.931 \\
\hline Male & 49 & 54 & & & 49 & 54 & & \\
\hline Female & 25 & 22 & & & 22 & 25 & & \\
\hline Bormann type & & & 4.743 & 0.029 & & & 0.018 & 0.894 \\
\hline 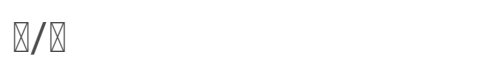 & 32 & 20 & & & 25 & 27 & & \\
\hline 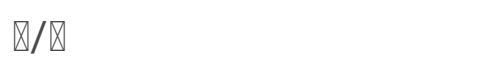 & 42 & 56 & & & 46 & 52 & & \\
\hline Tumor size(cm) & & & 0.455 & 0.500 & & & 0.318 & 0.573 \\
\hline$<5$ & 43 & 40 & & & 41 & 42 & & \\
\hline$\geq 5$ & 31 & 36 & & & 30 & 37 & & \\
\hline Lauren's classification & & & 5.489 & 0.064 & & & 2.263 & 0.323 \\
\hline Intestinal & 17 & 14 & & & 12 & 19 & & \\
\hline Diffuse & 40 & 31 & & & 38 & 33 & & \\
\hline Mixed & 17 & 31 & & & 21 & 27 & & \\
\hline Differentiation & & & 0.218 & 0.641 & & & 0.040 & 0.841 \\
\hline Well/Moderate & 31 & 29 & & & 29 & 31 & & \\
\hline Poor & 43 & 47 & & & 42 & 48 & & \\
\hline Histological type & & & 2.599 & 0.107 & & & 3.071 & 0.080 \\
\hline Adenocarcinoma & 66 & 73 & & & 63 & 76 & & \\
\hline Mucinous/Signet-ring cell & 8 & 3 & & & 8 & 3 & & \\
\hline Depth of tumor invasion & & & 11.786 & 0.001 & & & 4.192 & 0.041 \\
\hline
\end{tabular}




\begin{tabular}{|c|c|c|c|c|c|c|c|c|}
\hline \multirow{2}{*}{$\begin{array}{l}\text { Clinicopathological } \\
\text { parameters }\end{array}$} & \multicolumn{4}{|c|}{ P4HB expression } & \multicolumn{4}{|c|}{ GRP78 expression } \\
\hline & Low & High & $x^{2}$ & $\begin{array}{l}P \\
\text { value }\end{array}$ & Low & High & $x^{2}$ & $\begin{array}{l}P \\
\text { value }\end{array}$ \\
\hline T1/T2/T3 & 61 & 43 & & & 55 & 49 & & \\
\hline $\mathrm{T} 4$ & 13 & 33 & & & 16 & 30 & & \\
\hline Lymph node metastasis & & & 5.561 & 0.018 & & & 4.092 & 0.043 \\
\hline Negative & 32 & 19 & & & 30 & 21 & & \\
\hline Positive & 42 & 57 & & & 41 & 58 & & \\
\hline TNM stage & & & 3.209 & 0.073 & & & 0.914 & 0.339 \\
\hline 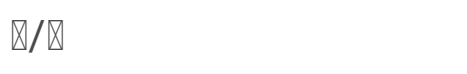 & 41 & 31 & & & 37 & 35 & & \\
\hline 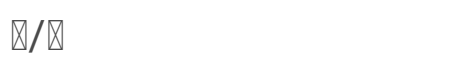 & 33 & 45 & & & 34 & 44 & & \\
\hline Vessel invasion & & & 0.064 & 0.800 & & & 0.609 & 0.435 \\
\hline Negative & 53 & 53 & & & 48 & 58 & & \\
\hline Positive & 21 & 23 & & & 23 & 21 & & \\
\hline Perineural invasion & & & 0.080 & 0.777 & & & 0.028 & 0.867 \\
\hline Negative & 56 & 59 & & & 54 & 61 & & \\
\hline Positive & 18 & 17 & & & 17 & 18 & & \\
\hline $\begin{array}{l}\text { Postoperative adjuvant } \\
\text { chemotherapy }\end{array}$ & & & 7.055 & 0.008 & & & 0.334 & 0.563 \\
\hline No & 17 & 33 & & & 22 & 28 & & \\
\hline Yes & 57 & 43 & & & 59 & 51 & & \\
\hline GRP78 expression & & & 34.562 & $<.001$ & & & & \\
\hline Low & 53 & 18 & & & & & & \\
\hline High & 21 & 58 & & & & & & \\
\hline
\end{tabular}

\section{Bioinformatics analyses of P4HB and GRP78 expression in $\mathrm{GC}$}

We explored TCGA-STAD (stomach adenocarcinoma) data by GEPIA and analyzed of extracted GC cell lines data (amount to 37 lines) from CCLE successively. It turned out P4HB mRNA was also positively 
correlated with GRP78 in both GC tissue and cell databases $(R=0.61, P<0.001$, and $R=0.356, P=0.031$, respectively) (sFigure 1B and 1C), in accordance with the correlation of proteins. Then we performed GO and KEGG analysis of these two genes. The top 5 items of each $G 0$ term was shown in sFigure 1D. Only items involve with both two genes were taken into account. Finally, protein folding and response to ER stress were discovered in biological process (BP) term, ER chaperone complex, melanosome, pigment granule, ER-Golgi intermediate compartment and ER lumen were discovered in cellular component (CC) term, together with only cell adhesion molecule binding was discovered in molecular function (MF) term. KEGG pathway analysis showed both two genes were enriched in the pathway "protein processing in ER" (sFigure 1E). As the occurrence and development of GC was most likely related to ER stress and UPR, we made further efforts to search correlation of P4HB or HSPA5 with genes within UPR, originated from the "protein processing in ER" KEGG pathway map (hsa04141), by explore TCGA-STAD cohort in GEPIA (sFigure 2-3). Except for P4HB and HSPA5 both positively correlated with typical genes within UPR from STAD (Table 2), correlation coefficients of most upstream genes were higher than downstream genes, as shown in sFigure $1 \mathrm{~F}$ and $1 \mathrm{G}$. 
Table 2

Correlation between P4HB/HSPA5 with genes in UPR pathways from TCGA-STAD cohort

\begin{tabular}{|c|c|c|c|c|c|c|}
\hline \multirow[t]{2}{*}{ Pathway } & \multirow{2}{*}{$\begin{array}{l}\text { Name in } \\
\text { pathway }\end{array}$} & \multirow{2}{*}{$\begin{array}{l}\text { Typical encoded } \\
\text { gene }\end{array}$} & \multicolumn{2}{|c|}{ P4HB } & \multicolumn{2}{|c|}{ HSPA5 } \\
\hline & & & $\mathbf{R}$ & $P$ value & $\mathbf{R}$ & $P$ value \\
\hline \multicolumn{7}{|l|}{$\begin{array}{l}\text { PERK } \\
\text { pathway }\end{array}$} \\
\hline & PERK & EIF2AK3 & 0.48 & $<0.001$ & 0.52 & $<0.001$ \\
\hline & elF1a & EIF2S1 & 0.41 & $<0.001$ & 0.53 & $<0.001$ \\
\hline & ATF4 & ATF4 & 0.19 & $<0.001$ & 0.22 & $<0.001$ \\
\hline & GADD34 & PPP1R15A & 0.22 & $<0.001$ & 0.30 & $<0.001$ \\
\hline & NRF2 & NFE2L2 & 0.27 & $<0.001$ & 0.31 & $<0.001$ \\
\hline \multicolumn{7}{|c|}{ ATF6 pathway } \\
\hline & ATF6 & ATF6 & 0.35 & $<0.001$ & 0.52 & $<0.001$ \\
\hline & S1P & MBTPS1 & 0.30 & $<0.001$ & 0.41 & $<0.001$ \\
\hline & S2P & MBTPS2 & 0.29 & $<0.001$ & 0.41 & $<0.001$ \\
\hline & WFS1 & WFS1 & 0.25 & $<0.001$ & 0.18 & $<0.001$ \\
\hline \multicolumn{7}{|c|}{ IRE1 pathway } \\
\hline & IRE1 & ERN1 & 0.47 & $<0.001$ & 0.29 & $<0.001$ \\
\hline & TRAF2 & TRAF2 & 0.41 & $<0.001$ & 0.41 & $<0.001$ \\
\hline & ASK1 & MAP3K5 & 0.20 & $<0.001$ & 0.31 & $<0.001$ \\
\hline & MKK7 & MAP2K7 & 0.22 & $<0.001$ & 0.11 & 0.021 \\
\hline & JNK & MAPK8 & 0.31 & $<0.001$ & 0.42 & $<0.001$ \\
\hline & XBP & XBP1 & 0.48 & $<0.001$ & 0.38 & $<0.001$ \\
\hline
\end{tabular}

\section{Association of P4HB and GRP78 expression with OS in GC}

We applied Kaplan-Meier survival analysis and log-rank test to compare OS according to P4HB and GRP78 expression. The results revealed that high expression of P4HB (Fig. 2A) and GRP78 (Fig. 2B) alone was related to an obvious shorter $\mathrm{OS}(\mathrm{P}<0.001$ and $\mathrm{P}=0.012$, respectively). Further stratification 
analysis showed that the prognosis was the best when P4HB and GRP78 were both low expressed, the worst prognosis was correlated with high expression of both P4HB and GRP78, while the prognosis was in between when one of P4HB and GRP78 was high expressed (Fig. 2C, P = 0.002). When analyzing of coexpression of P4HB and GRP78, OS of both or one high expression was sharp lower than that of both low expression (Fig. 2D, $\mathrm{P}=0.002$ ).

Since P4HB and GRP78 were both reported to be associated with chemotherapy, we also explored the prognosis of fluorouracil-based postoperative adjuvant chemotherapy together with co-expression of P4HB and GRP78, which contained both variables might more precise. We observed the prognosis of group with postoperative adjuvant chemotherapy was better than the other group (Fig. 2E, $P=0.011$ ). High level of P4HB and/or GRP78 exhibited significant worse in the former group (Fig. 2F, $P=0.007$ ), but no prognostic significance in the latter (Fig. $2 G, P=0.231$ ). Once the group with postoperative adjuvant chemotherapy was further divided according to TNM stage, it exhibited no prognostic significance in

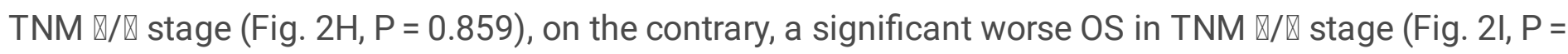
0.004). This indicates that GC patients in TNM $\otimes / \otimes$ stage may not benefit from postoperative adjuvant chemotherapy if P4HB and/or GRP78 high expressed.

\section{Construction of a nomogram based on stepwise Cox regression analysis}

Univariate analyses were performed for clinicopathological characteristics to estimate postoperative survival in our study group. According to the Table 3 , we observed that OS was significantly correlated with Bormann type $(P=0.004)$, tumor size $(P=0.028)$, differentiation $(P=0.004)$, depth of tumor invasion $(P<0.001)$, lymph node metastasis $(P<0.001)$, TNM stage $(P<0.001)$, postoperative adjuvant chemotherapy $(P=0.012)$, P4HB expression $(P=0.001)$, GRP78 expression $(P=0.013), P 4 H B$ and GRP78 co-expression $(P=0.002)$. These statistically significant variables were subsequent recruited to forward stepwise COX analysis. The result showed that differentiation $(P=0.020, H R=1.846,95 \% \mathrm{Cl}: 1.099-$ 3.101), TNM stage $(P<0.001, H R=3.124,95 \% \mathrm{Cl}: 1.870-5.217)$, postoperative adjuvant chemotherapy $(P=0.005, H R=0.507,95 \% \mathrm{Cl}: 0.317-0.810)$ and $P 4 H B$ and $G R P 78$ co-expression $(P=0.002, H R=2.304$, $95 \% \mathrm{Cl}: 1.355-3.915)$ were independent prognostic factors in GC patients (Table 3 ). Then four independent prognostic factors were included to construct a nomogram for predicting OS probability of GC patients (Fig. 3A). Harrell's C-index for nomogram was 0.733 (95\% Cl: $0.680-0.786$ ). And calibration plots for nomogram were close to the 45-degree line (the perfect calibration model) at 3-year and 5-year (Fig. 3B and 3C), indicated the model was well calibrated. 
Table 3

Univariate and multivariate analyses of prognostic characteristics for overall survival in patients with gastric cancer

\begin{tabular}{|c|c|c|c|c|c|c|}
\hline \multirow[t]{2}{*}{ Variables } & \multicolumn{3}{|c|}{ Univariate analysis } & \multicolumn{3}{|c|}{ Multivariate analysis } \\
\hline & HR & $(95 \% \mathrm{Cl})$ & $\begin{array}{l}P \\
\text { value }\end{array}$ & HR & $(95 \% \mathrm{Cl})$ & $\begin{array}{l}P \\
\text { value }\end{array}$ \\
\hline \multicolumn{7}{|l|}{ Age(year) } \\
\hline$\geq 65$ vs. $<65$ & 1.246 & $\begin{array}{l}0.791- \\
1.963\end{array}$ & 0.343 & & & \\
\hline \multicolumn{7}{|l|}{ Gender } \\
\hline Female vs. Male & 1.081 & $\begin{array}{l}0.665- \\
1.756\end{array}$ & 0.754 & & & \\
\hline \multicolumn{7}{|l|}{ Bormann type } \\
\hline$\nabla / \nabla$ vs. $\otimes / \nabla$ & 2.142 & $\begin{array}{l}1.271- \\
3.609\end{array}$ & 0.004 & & & \\
\hline \multicolumn{7}{|l|}{ Tumor size(cm) } \\
\hline$\geq 5$ vs. $<5$ & 1.662 & $\begin{array}{l}1.056- \\
2.616\end{array}$ & 0.028 & & & \\
\hline Lauren's classification & & & 0.214 & & & \\
\hline Intestinal & 1.000 & Reference & & & & \\
\hline Diffuse & 1.818 & $\begin{array}{l}0.932- \\
3.545\end{array}$ & 0.079 & & & \\
\hline Mixed & 1.613 & $\begin{array}{l}0.790- \\
3.293\end{array}$ & 0.190 & & & \\
\hline \multicolumn{7}{|l|}{ Differentiation } \\
\hline Poor vs. Well/Moderate & 2.111 & $\begin{array}{l}1.274- \\
3.497\end{array}$ & 0.004 & 1.846 & $\begin{array}{l}1.099- \\
3.101\end{array}$ & 0.020 \\
\hline \multicolumn{7}{|l|}{ Histological type } \\
\hline Mucinous/Signet-ring cell & 0.592 & $\begin{array}{l}0.216- \\
1.622\end{array}$ & 0.308 & & & \\
\hline \multicolumn{7}{|l|}{ vs. Adenocarcinoma } \\
\hline \multicolumn{7}{|l|}{ Depth of tumor invasion } \\
\hline T4 vs. T1/T2/T3 & 2.536 & $\begin{array}{l}1.601- \\
4.019\end{array}$ & $<_{0.001}$ & & & \\
\hline
\end{tabular}




\begin{tabular}{|c|c|c|c|c|c|c|}
\hline \multirow[t]{2}{*}{ Variables } & \multicolumn{3}{|c|}{ Univariate analysis } & \multicolumn{3}{|c|}{ Multivariate analysis } \\
\hline & HR & $(95 \% \mathrm{Cl})$ & $\begin{array}{l}P \\
\text { value }\end{array}$ & HR & $(95 \% \mathrm{Cl})$ & $\begin{array}{l}P \\
\text { value }\end{array}$ \\
\hline Positive vs. Negative & 3.824 & $\begin{array}{l}2.060- \\
7.101\end{array}$ & $<.001$ & & & \\
\hline \multicolumn{7}{|l|}{ TNM stage } \\
\hline \/\ vs. $\nabla / \bigotimes$ & 3.200 & $\begin{array}{l}1.942- \\
5.275\end{array}$ & $\begin{array}{l}< \\
0.001\end{array}$ & 3.124 & $\begin{array}{l}1.870- \\
5.217\end{array}$ & $\begin{array}{l}< \\
0.001\end{array}$ \\
\hline \multicolumn{7}{|l|}{ Vessel invasion } \\
\hline Positive vs. Negative & 1.385 & $\begin{array}{l}0.861- \\
2.229\end{array}$ & 0.180 & & & \\
\hline \multicolumn{7}{|l|}{ Perineural invasion } \\
\hline Positive vs. Negative & 1.279 & $\begin{array}{l}0.766^{-} \\
2.135\end{array}$ & 0.346 & & & \\
\hline \multicolumn{7}{|l|}{$\begin{array}{l}\text { Postoperative adjuvant } \\
\text { chemotherapy }\end{array}$} \\
\hline Yes vs. No & 0.556 & $\begin{array}{l}0.351- \\
0.879\end{array}$ & 0.012 & 0.507 & $\begin{array}{l}0.317- \\
0.810\end{array}$ & 0.005 \\
\hline \multicolumn{7}{|l|}{ P4HB expression } \\
\hline High vs. Low & 2.266 & $\begin{array}{l}1.414- \\
3.630\end{array}$ & 0.001 & & & \\
\hline \multicolumn{7}{|l|}{ GRP78 expression } \\
\hline High vs. Low & 1.799 & $\begin{array}{l}1.130- \\
2.862\end{array}$ & 0.013 & & & \\
\hline \multicolumn{7}{|c|}{ P4HB and GRP78 co-expression } \\
\hline Both/one high vs. Both low & 2.260 & $\begin{array}{l}1.338- \\
3.817\end{array}$ & 0.002 & 2.304 & $\begin{array}{l}1.355- \\
3.915\end{array}$ & 0.002 \\
\hline
\end{tabular}

\section{Evaluate the nomogram and its clinical value with TNM stage}

To evaluate the discrimination ability and clinical uesfulness of the nomogram, we compared it with widely clinical used TNM stage. From Fig. 4A and 4B, we found areas under ROC (receiver operating characteristic) curve (AUROCs) of the 3-year and 5-year OS probability for nomogram model were 0.790 and 0.760 , respectively, much better than that for TNM stage ( 0.684 and 0.671 , respectively). Since DCA has superiorities over AUROC for estimating alternative prognostic strategies, we performed the 3-year 
and 5-year DCA curves individually for the nomogram and TNM stage. As shown in Fig. 4C and 4D, compared to TNM stage, even at a very small threshold probability, the nomogram offered a high net benefit in 3-year and 5-year, respectively.

\section{Discussion}

ER is not only high in calcium-dependent molecular chaperones such as GRP78 for helping stabilize protein-folding intermediates, also has an oxidative environment for the formation of disulphide bonds with the aid of PDI and for the proper folding of proteins [23, 24]. Under non-stressed conditions, GRP78 combines with three UPR sensors (PERK, ATF6 and IRE1) keeping them inactive. Once ER stress occurs, GRP78 preferentially binds to the unfolded proteins, dissociates from the sensors, resulting in activation of the UPR transducers $[6,25]$. The outcome of it involves in protein folding and transporting, as well as increasing unfolded protein clearance through pathways such as ERassociated degradation (ERAD) and autophagy. Upon unresolved stress, cells enter apoptosis [8].

PDI family is a part of the thioredoxin (TRX) superfamily, mainly act as catalysts for the formatting and rearranging S-S bonds, also can function as chaperones involved in ERAD [26], playing an important role in the maintenance and regulation of proteostasis. P4HB, also known as PDIA1 (usually referred as PDI), as the archetype PDI protein, the chaperone activity of who is independent of catalytic activity, while the former is vital for many client proteins [27]. There is sufficient evidence supporting that PDIA1 high expressed in a wide variety of cancers tissues [9-11,24] and cancer cells [28]. And the upregulated PDIA1 is closely correlated with cancer metastasis and invasiveness [27].

GRP78 is encoded by HSPA5, as the most abundant protein of heat shock protein-70 (Hsp70) family, mainly exits in ER lumen, corrects the folding and assembly, as well as prevents the transport of misfolded proteins or protein subunits $[29,30]$. GRP78 works as a molecular chaperone in cells, its high expression is crucial for proliferation, invasion, metastasis of many cancers, proves to be correlated with tumor resistance, recurrence, and a low OS [17, 18, 31-33]. With the help of GRP78, tumor cells can organize the stimulation of processes including macroautophagy, combat reactive oxygen species (ROS), and activate pro-survival signaling pathways [29]. Thus, GRP78 will be a useful prognostic biomarker and a promising target for cancer.

P4HB and GRP78 are both ER chaperone proteins involved in UPR, and represent for poor prognosis of various cancers from the above mentioned. However, rare research pays attention to the correlation of them, especially in cancer. An early study shows that BiP and PDI perform synergistically in vitro in the oxidative folding of antibodies, BiP bind to the unfolded polypeptide chains to keep them in a conformation where cysteine residues are accessible for PDI [34]. Different from P4HB negatively correlated with GRP78 in hepatocellular cancer in vitro [19], we confirmed that P4HB and GRP78 showed a positive correlation in protein (from our cohort) and mRNA (from the databases) levels, with disease progress, the two proteins might more closely related since the correlation coefficients were significantly elevated in tumor size $\geq 5 \mathrm{~cm}, \mathrm{TNM} \otimes / \nabla$ stage and positive vessel invasion subgroups. Together with 
single or combined high expression of P4HB and GRP78 represented for poor prognosis in GC. The specific mechanisms need further exploration.

A lot of studies have shown in a wide range of primary tumors, there exists a sustained and high-level activation of all three branches within UPR [35]. From bioinformatics analyses, we also found P4HB and GRP78 might participate in UPR, since they positively correlated with all typical genes within UPR, as well as correlation coefficients of most upstream genes were higher than downstream genes. UPR may act as a key driver in resistance to chemotherapy [36,37]. A new encouraging research finds that activation of the PERK branch with UPR is required for colon cancer cells surviving from treatment of 5-fluorouracil, the usage of PERK inhibitor synergizes with 5-FU could suppress the growth of colon cancer cells in vivo [38]. It may explain in fluorouracil-based postoperative adjuvant chemotherapy subgroup, GC patients with a high co-expression of P4HB and GRP78 had a shorter OS of our cohort. When stratified by TNM stage,

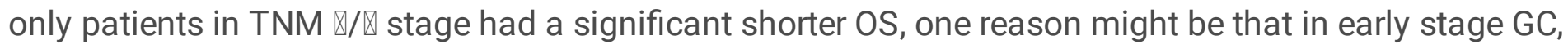
the positive effects from chemotherapy could cancel out the negative effects from UPR, while in advanced GC, the negative effects probably exceeded.

Our nomogram integrated co-expression of P4HB and GRP78 with other prognostic variables, was a simple visualized tool for predicting OS after surgery of patients with OS. Comparing to TNM stage, the discrimination ability and clinical usefulness of the nomogram was much better through AUROCs and DCA curves of 3-year and 5-year, may be great valuable for clinic.

There still remain several limitations in our study. Firstly, this is a retrospective study of small sample group of GC patients, exists the statistical limitations. Secondly, further researches are required to confirm the correlation and regulation mechanisms of P4HB and GRP78 expression of GC in vitro and in vivo. Finally, it needs an independent external cohort to verify our research findings.

In conclusion, we revealed that P4HB expression is positively correlated with GRP78 expression, the correlationship may closer in advanced GC; P4HB and GRP78 may involve in UPR of GC from bioinformatics analyses; high co-expression of P4HB and GRP78 predicts a shorter OS with fluorouracilbased postoperative adjuvant chemotherapy in the advanced stage; co-expression of P4HB and GRP78 can independently predict unfavorable OS for GC patients; the prognostic nomogram based on stepwise Cox regression model is much better than TNM stage in the discrimination ability and clinical usefulness.

\section{Declarations}

\section{Acknowledgements}

We thank all the contributors for this work.

\section{Authors' contributions}


Zhang CF and Zhao WJ performed the majority of experiments and wrote the manuscript; Yang $\mathrm{L}$ and Chen XD designed and supervised the study; Zhang XS and Wu TY assisted in conducting the IHC assays; Zhang XL and Xu WW collected and analyzed the data; Song L helped to correct the manuscript.

\section{Funding}

This work was supported by Natural Science Foundation of Jiangsu Province(BK20191208);Basic Science Research Project of Nantong(JC2018031) ; the Youth Science and Research Foundation of Nantong Health Commission (QA2019025).

\section{Availability of data and materials}

All data and material are available from the corresponding author.

\section{Ethics approval and consent to participate}

This research was reviewed and approved by the Ethics Committee of Affiliated Tumor Hospital of Nantong University

\section{Consent for publication}

The publication of this manuscript has been approved by all authors.

\section{Competing interests}

The authors declare that they have no competing interests.

\section{References}

1. Bray F, Ferlay J, Soerjomataram I, Siegel RL, Torre LA, Jemal A: Global cancer statistics 2018: GLOBOCAN estimates of incidence and mortality worldwide for 36 cancers in 185 countries. $C A$ Cancer J Clin 2018, 68(6):394-424.

2. Van Cutsem E, Sagaert X, Topal B, Haustermans K, Prenen H: Gastric cancer. The Lancet 2016, 388(10060):2654-2664.

3. Allemani C, Matsuda T, Di Carlo V, Harewood R, Matz M, Nikšić M, Bonaventure A, Valkov M, Johnson $\mathrm{CJ}$, Estève $\mathrm{J}$ et al: Global surveillance of trends in cancer survival 2000-14 (CONCORD-3): analysis of individual records for 37513025 patients diagnosed with one of 18 cancers from 322 populationbased registries in 71 countries. The Lancet 2018, 391(10125):1023-1075. 
4. Setia N, Agoston AT, Han HS, Mullen JT, Duda DG, Clark JW, Deshpande V, Mino-Kenudson M, Srivastava A, Lennerz JK et al: A protein and mRNA expression-based classification of gastric cancer. Mod Pathol 2016, 29(7):772-784.

5. Jiang Y, Zhang Q, Hu Y, Li T, Yu J, Zhao L, Ye G, Deng H, Mou T, Cai S et al: ImmunoScore Signature: A Prognostic and Predictive Tool in Gastric Cancer. Ann Surg 2018, 267(3):504-513.

6. Tameire F, Verginadis, II, Koumenis C: Cell intrinsic and extrinsic activators of the unfolded protein response in cancer: Mechanisms and targets for therapy. Semin Cancer Biol 2015, 33:3-15.

7. Kranz P, Neumann F, Wolf A, Classen F, Pompsch M, Ocklenburg T, Baumann J, Janke K, Baumann M, Goepelt $\mathrm{K}$ et al: $\mathrm{PDI}$ is an essential redox-sensitive activator of PERK during the unfolded protein response (UPR). Cell Death Dis 2017, 8(8):e2986.

8. Wang $M$, Kaufman RJ: The impact of the endoplasmic reticulum protein-folding environment on cancer development. Nat Rev Cancer 2014, 14(9):581-597.

9. Zou H, Wen C, Peng Z, Shao Y, Hu L, Li S, Li C, Zhou HH: P4HB and PDIA3 are associated with tumor progression and therapeutic outcome of diffuse gliomas. Oncol Rep 2018, 39(2):501-510.

10. Wang SM, Lin LZ, Zhou DH, Zhou JX, Xiong SQ: Expression of prolyl 4-hydroxylase beta-polypeptide in non-small cell lung cancer treated with Chinese medicines. Chin J Integr Med 2015, 21(9):689-696.

11. Zhou Y, Yang J, Zhang Q, Xu Q, Lu L, Wang J, Xia W: P4HB knockdown induces human HT29 colon cancer cell apoptosis through the generation of reactive oxygen species and inactivation of STAT3 signaling. Mol Med Rep 2019, 19(1):231-237.

12. Xu S, Sankar S, Neamati N: Protein disulfide isomerase: a promising target for cancer therapy. Drug Discov Today 2014, 19(3):222-240.

13. Liu Z, Wang Y, Wang Y, Dong W, Xia X, Song E, Song Y: Effect of Subcellular Translocation of Protein Disulfide Isomerase on Tetrachlorobenzoquinone-Induced Signaling Shift from Endoplasmic Reticulum Stress to Apoptosis. Chem Res Toxico/ 2017, 30(10):1804-1814.

14. Kullmann M, Kalayda GV, Hellwig M, Kotz S, Hilger RA, Metzger S, Jaehde U: Assessing the contribution of the two protein disulfide isomerases PDIA1 and PDIA3 to cisplatin resistance. $J$ Inorg Biochem 2015, 153:247-252.

15. Zhang J, Wu Y, Lin YH, Guo S, Ning PF, Zheng ZC, Wang Y, Zhao Y: Prognostic value of hypoxiainducible factor-1 alpha and prolyl 4-hydroxylase beta polypeptide overexpression in gastric cancer. World J Gastroenterol 2018, 24(22):2381-2391.

16. Kwon D, Koh J, Kim S, Go H, Min HS, Kim YA, Kim DK, Jeon YK, Chung DH: Overexpression of endoplasmic reticulum stress-related proteins, XBP1s and GRP78, predicts poor prognosis in pulmonary adenocarcinoma. Lung Cancer 2018, 122:131-137.

17. Nagelkerke A, Bussink J, Sweep FC, Span PN: The unfolded protein response as a target for cancer therapy. Biochim Biophys Acta 2014, 1846(2):277-284.

18. Gifford JB, Hill R: GRP78 Influences Chemoresistance and Prognosis in Cancer. Curr Drug Targets 2018, 19(6):701-708. 
19. Xia W, Zhuang J, Wang G, Ni J, Wang J, Ye Y: P4HB promotes HCC tumorigenesis through downregulation of GRP78 and subsequent upregulation of epithelial-to-mesenchymal transition. Oncotarget 2017, 8(5):8512-8521.

20. Li L, Cui Y, Ji JF, Jiang WG: Clinical Correlation Between WISP2 and beta-Catenin in Gastric Cancer. Anticancer Res 2017, 37(8):4469-4473.

21. Tang Z, Li C, Kang B, Gao G, Li C, Zhang Z: GEPIA: a web server for cancer and normal gene expression profiling and interactive analyses. Nucleic Acids Res 2017, 45(W1):W98-W102.

22. Barretina J, Caponigro G, Stransky N, Venkatesan K, Margolin AA, Kim S, Wilson CJ, Lehar J, Kryukov GV, Sonkin D et al: The Cancer Cell Line Encyclopedia enables predictive modelling of anticancer drug sensitivity. Nature 2012, 483(7391):603-607.

23. Kim I, Xu W, Reed JC: Cell death and endoplasmic reticulum stress: disease relevance and therapeutic opportunities. Nat Rev Drug Discov 2008, 7(12):1013-1030.

24. Maattanen P, Gehring K, Bergeron JJ, Thomas DY: Protein quality control in the ER: the recognition of misfolded proteins. Semin Cell Dev Biol 2010, 21(5):500-511.

25. Lai E, Teodoro T, Volchuk A: Endoplasmic Reticulum Stress: Signaling the Unfolded Protein Response. Physiology 2007, 22:193-201.

26. Hatahet $F$, Ruddock LW: Substrate recognition by the protein disulfide isomerases. FEBS Journal 2007, 274(20):5223-5234.

27. Lee $\mathrm{E}$, Lee $\mathrm{DH}$ : Emerging roles of protein disulfide isomerase in cancer. $B M B \operatorname{Rep} 2017,50(8): 401$ 410.

28. Shin BK, Wang H, Yim AM, Le Naour F, Brichory F, Jang JH, Zhao R, Puravs E, Tra J, Michael CW et al: Global profiling of the cell surface proteome of cancer cells uncovers an abundance of proteins with chaperone function. J Biol Chem 2003, 278(9):7607-7616.

29. Casas C: GRP78 at the Centre of the Stage in Cancer and Neuroprotection. Frontiers in Neuroscience 2017, 11.

30. Ibrahim IM, Abdelmalek DH, Elfiky AA: GRP78: A cell's response to stress. Life Sciences 2019, 226:156-163.

31. Guan M, Chen X, Ma Y, Tang L, Guan L, Ren X, Yu B, Zhang W, Su B: MDA-9 and GRP78 as potential diagnostic biomarkers for early detection of melanoma metastasis. Tumor Biology 2014, 36(4):29732982.

32. Mhaidat NM, Alzoubi KH, Almomani N, Khabour OF: Expression of glucose regulated protein 78 (GRP78) determines colorectal cancer response to chemotherapy. Cancer Biomark 2015, 15(2):197203.

33. Dai YJ, Qiu YB, Jiang R, Xu M, Liao LY, Chen GG, Liu ZM: Concomitant high expression of ERalpha36, GRP78 and GRP94 is associated with aggressive papillary thyroid cancer behavior. Cell Oncol (Dordr) 2018, 41(3):269-282. 
34. Mayer M, Kies U, Kammermeier R, Buchner J: BiP and PDI cooperate in the oxidative folding of antibodies in vitro. J Biol Chem 2000, 275(38):29421-29425.

35. Oakes SA, Papa FR: The role of endoplasmic reticulum stress in human pathology. Annu Rev Pathol 2015, 10:173-194.

36. Wang $Y$, Wang $K$, Jin Y, Sheng $X$ : Endoplasmic reticulum proteostasis control and gastric cancer. Cancer Lett 2019, 449:263-271.

37. Kim C, Kim B: Anti-Cancer Natural Products and Their Bioactive Compounds Inducing ER StressMediated Apoptosis: A Review. Nutrients 2018, 10(8).

38. Shi Z, Yu X, Yuan M, Lv W, Feng T, Bai R, Zhong H: Activation of the PERK-ATF4 pathway promotes chemo-resistance in colon cancer cells. Sci Rep 2019, 9(1):3210.

\section{Figures}

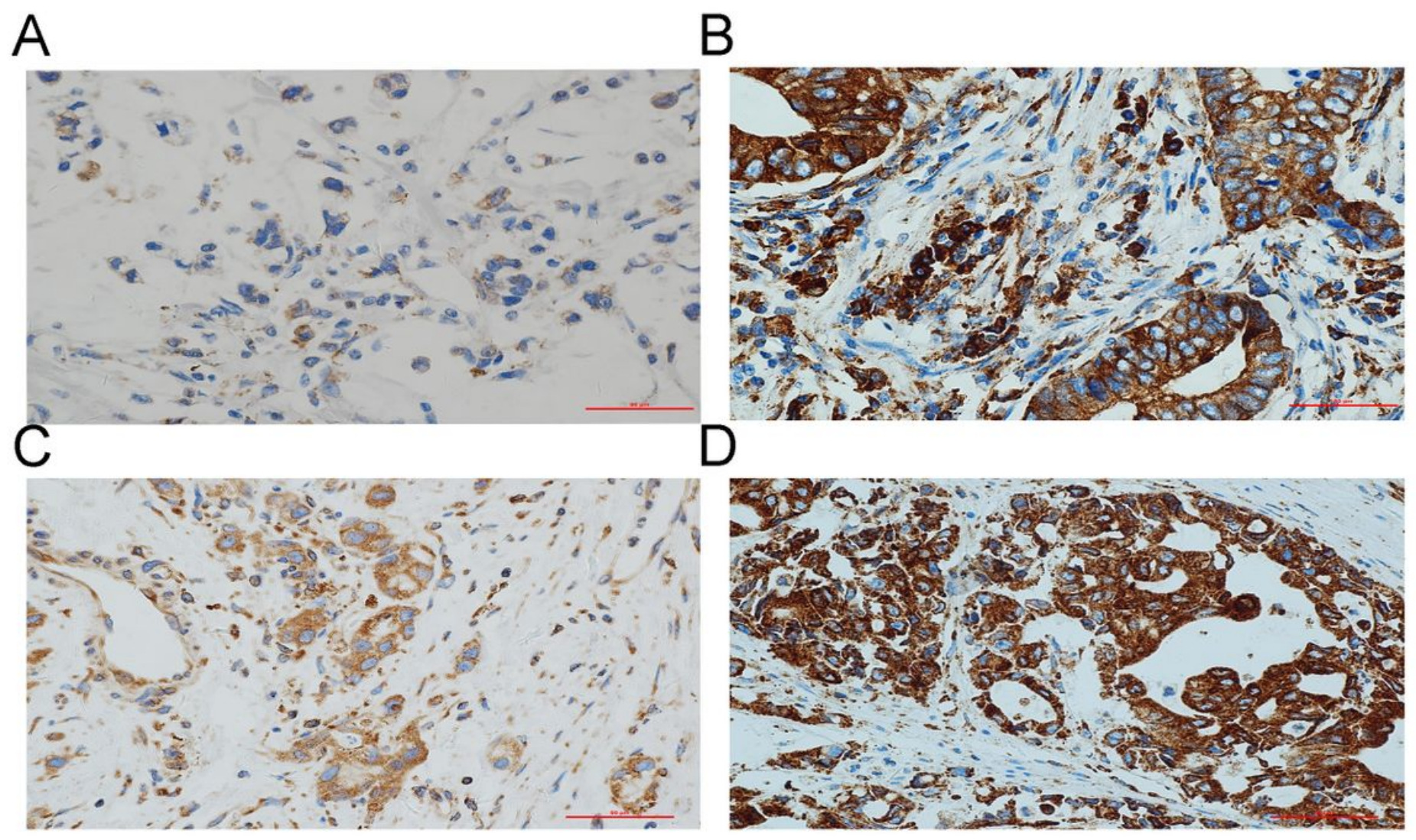

Figure 1

Representative immunohistochemical staining images of P4HB and GRP78 in gastric cancer (GC) tissues. A: Low expression of P4HB in the cytoplasm of a GC case.B: High expression of P4HB in the cytoplasm of a GC case. C: Low expression of GRP78 in the cytoplasm of a GC case. D: High expression of GRP78 in the cytoplasm of a GC case. Magnifications in all figures were $400 \times$. 
A

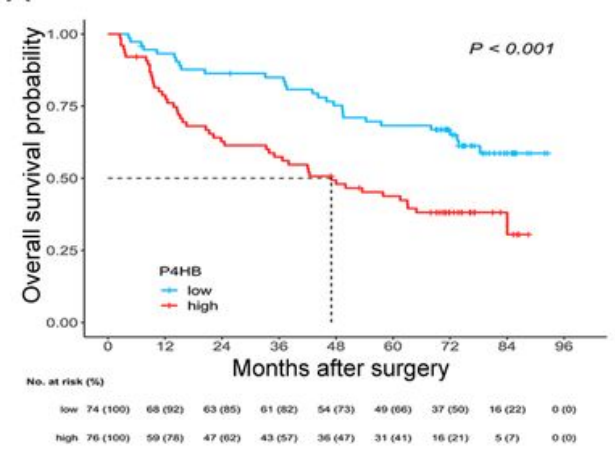

D

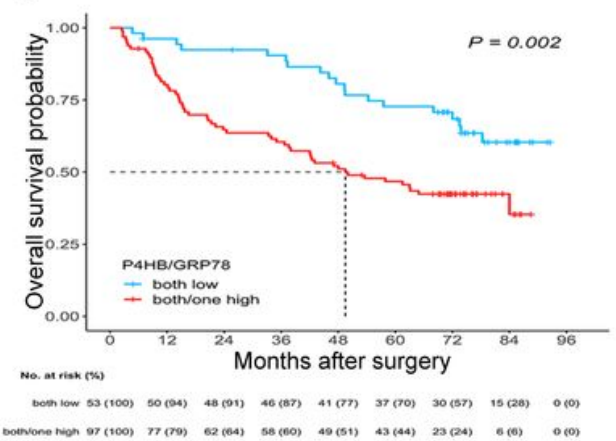

G

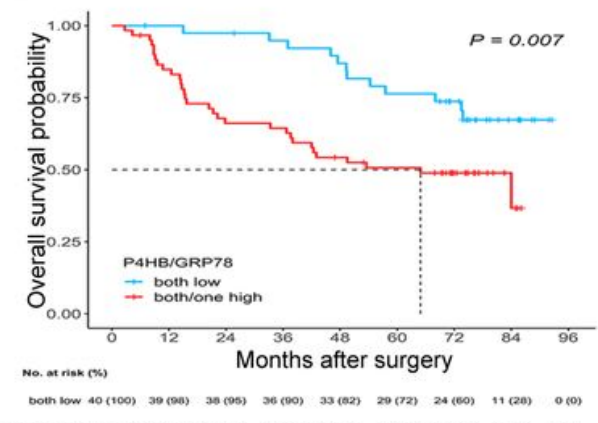

B

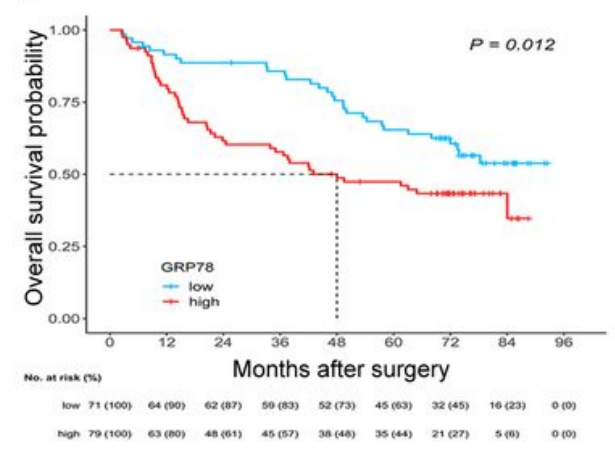

E

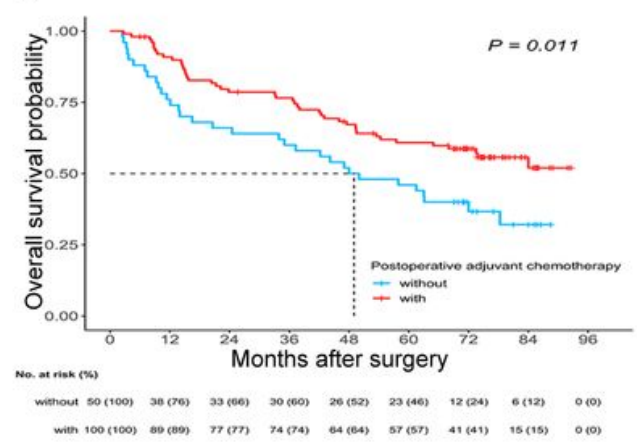

$\mathrm{H}$

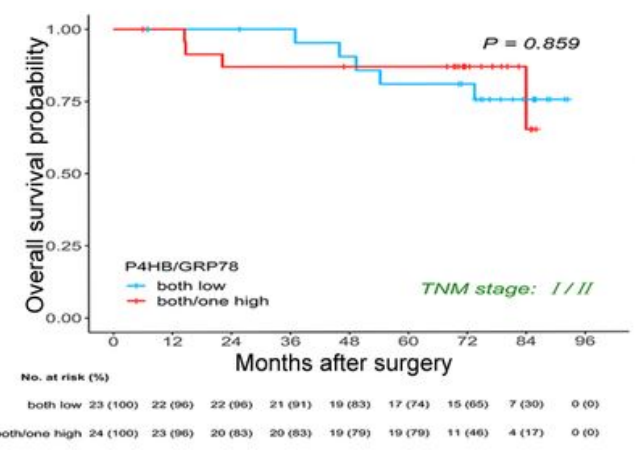

C

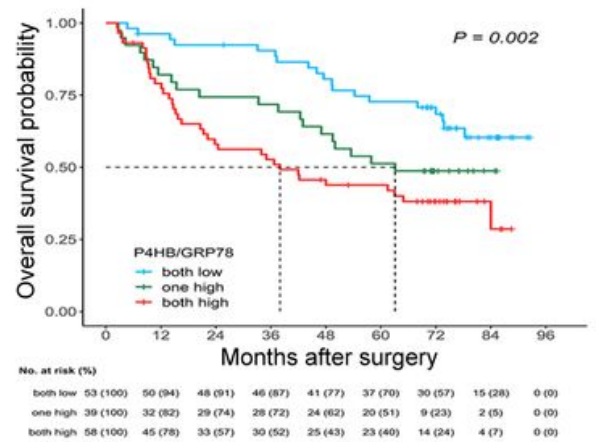

$\mathrm{F}$

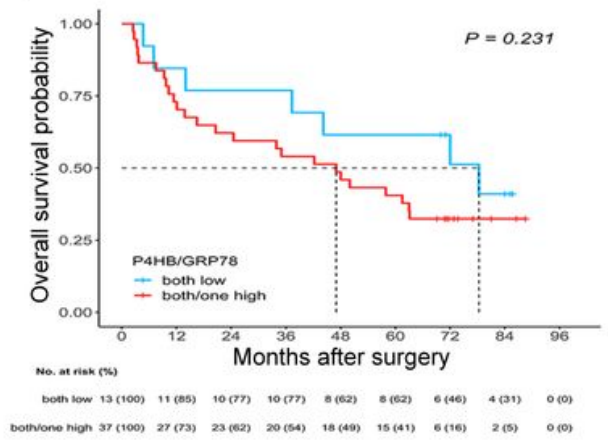

।

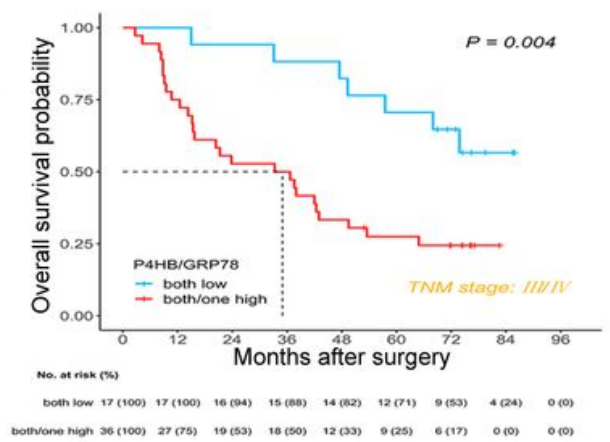

Figure 2

Kaplan-Meier analyses of OS in GC patients. A: Kaplan-Meier analysis of OS according to single P4HB expression. B: Kaplan-Meier analysis of OS according to single GRP78 expression. C and D: Kaplan-Meier analysis of OS according to combined P4HB and GRP78 expression. E: Kaplan-Meier analysis of OS according to postoperative adjuvant chemotherapy. F: Kaplan-Meier analysis of OS in patients without postoperative adjuvant chemotherapy according to co-expression of P4HB and GRP78. G: Kaplan-Meier analysis of OS in patients with postoperative adjuvant chemotherapy according to co-expression of $\mathrm{P} 4 \mathrm{HB}$

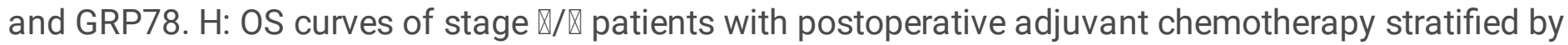
co-expression of P4HB and GRP78. I: OS curves of stage $\otimes / \otimes$ patients with postoperative adjuvant chemotherapy stratified by co-expression of P4HB and GRP78. 
A
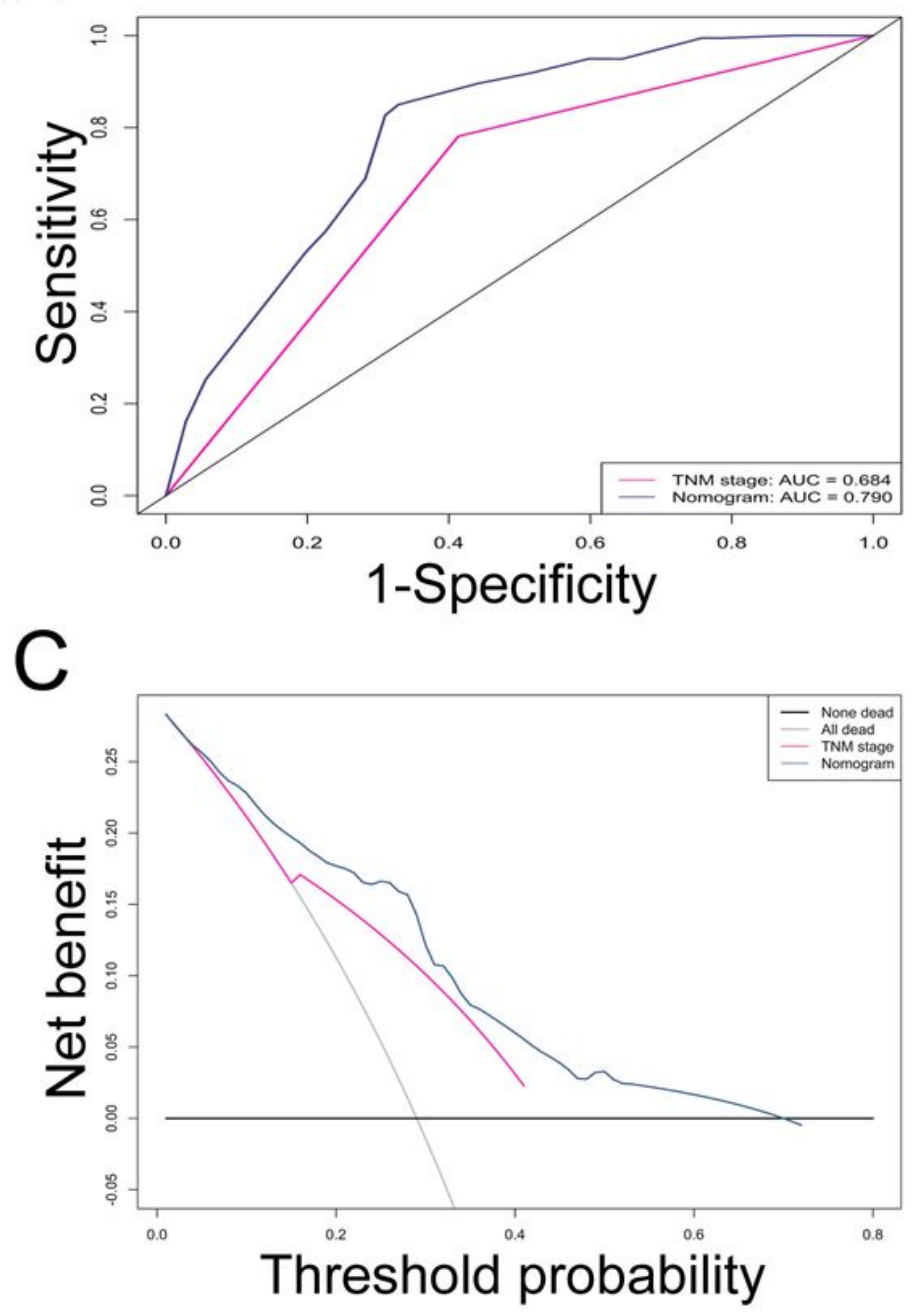
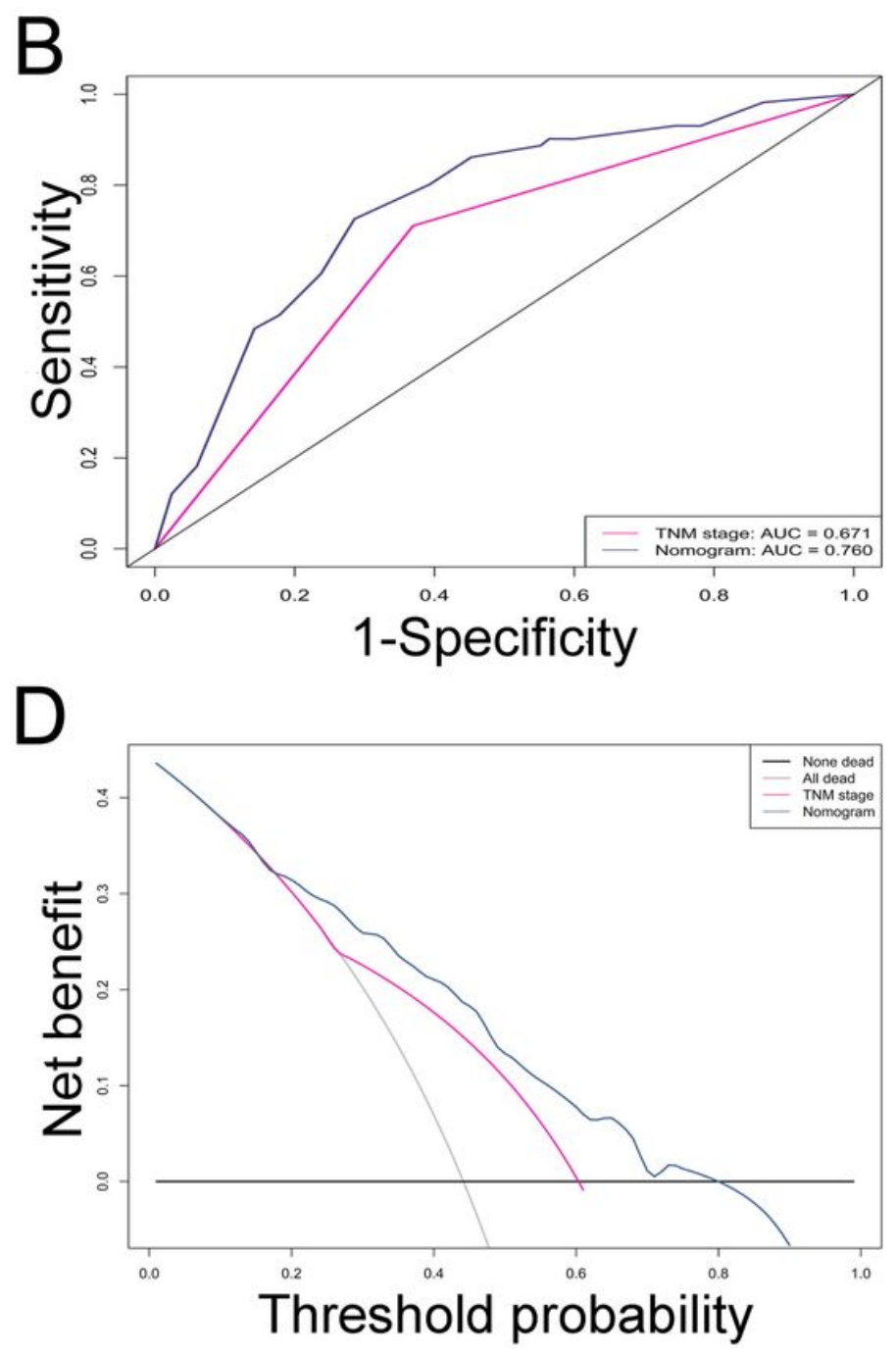

Figure 4

ROC and DCA curves of nomogram and TNM stage for OS prediction in GC patients. A: ROC curves for predicting 3-year OS; B: ROC curves for predicting 5-year OS; C: DCA curves for predicting 3-year OS; D: DCA curves for predicting 5-year OS.

\section{Supplementary Files}

This is a list of supplementary files associated with this preprint. Click to download.

- Sfigure3.tif

- Sfigure2.tif

- Sfigure1.tif

- supplementarymaterials.docx 